\title{
Growth of Matter Perturbations in an Interacting Dark Energy Scenario Emerging from Metric-Scalar-Torsion Couplings ${ }^{\dagger}$
}

\author{
Mohit Kumar Sharma * and Sourav Sur (D)
}

check for

updates

Citation: Sharma, M.K.; Sur, S Growth of Matter Perturbations in an Interacting Dark Energy Scenario Emerging from Metric-Scalar-Torsion Couplings. Phys. Sci. Forum 2021, 2, 51. https://doi.org/10.3390/ ECU2021-09293

Academic Editor: Lorenzo Iorio

Published: 22 February 2021

Publisher's Note: MDPI stays neutral with regard to jurisdictional claims in published maps and institutional affiliations.

Copyright: (c) 2021 by the authors. Licensee MDPI, Basel, Switzerland. This article is an open access article distributed under the terms and conditions of the Creative Commons Attribution (CC BY) license (https:// creativecommons.org/licenses/by/ $4.0 /)$.
Department of Physics and Astrophysics, University of Delhi, New Delhi 110 007, India; sourav@physics.du.ac.in or sourav.sur@gmail.com

* Correspondence: mr.mohit254@gmail.com

† Presented at the 1st Electronic Conference on Universe, 22-28 February 2021; Available online: https://ecu2021.sciforum.net/.

Abstract: We study the growth of linear matter density perturbations in a modified gravity approach of scalar field couplings with metric and torsion. In the equivalent scalar-tensor formulation, the matter fields in the Einstein frame interact as usual with an effective dark energy component, whose dynamics are presumably governed by a scalar field that sources a torsion mode. As a consequence, the matter density ceases to be self-conserved, thereby making an impact not only on the background cosmological evolution but also on the perturbative spectrum of the local inhomogeneities. In order to estimate the effect on the growth of the linear matter perturbations, with the least possible alteration of the standard parametric form of the growth factor, we resort to a suitable Taylor expansion of the corresponding exponent, known as the growth index, about the value of the cosmic scale factor at the present epoch. In particular, we obtain an appropriate fitting formula for the growth index in terms of the coupling function and the matter density parameter. While the overall parametric formulation of the growth factor is found to fit well with the latest redshift-space-distortion (RSD) and the observational Hubble $(\mathrm{OH})$ data at low redshifts, the fitting formula enables us to constrain the growth index to well within the concordant cosmological limits, thus ensuring the viability of the formalism.

Keywords: cosmological perturbations; dark energy theory; modified gravity; torsion; cosmology of theories beyond the SM

\section{Introduction}

The effect of the evolving dark energy (DE) on the rate of the large-scale structure (LSS) formation has been a prime area of investigation in modern cosmology, particularly from the point of view of asserting the characteristics of the respective DE component [1-5]. While the observations grossly favor such a component to be a cosmological constant $\Lambda$ [6-12], a stringent fine-tuning problem associated with the corresponding model, viz. $\Lambda \mathrm{CDM}$ (where CDM stands for cold dark matter), has prompted extensive explorations of a dynamically evolving DE from various perspectives. Moreover, certain observational results do provide some scope of a plausible dynamical DE evolution, albeit up to a significant degree of mildness. In this context, it is worth noting that, however mild the DE dynamics may be, at the standard Friedmann-Robertson-Walker (FRW) background cosmological level, there may be substantial effects of such dynamics on the spectrum of the linear matter density perturbations. Hence, the analysis of the observational data on the evolution of such perturbations, or the LSS growth data, is crucial for constraining dynamical DE models of all sorts.

Apart from the commonly known dynamical DE models involving scalar fields (such as quintessence, kessence, and so on [13-21]), a considerable interest has developed in recent years in the cosmological scenarios emerging from scalar-tensor equivalent modified 
gravity (MG) theories [22-26] that stretch beyond the standard principles of General Relativity (GR). Such scenarios are particularly useful for providing plausible resolutions to the issue of cosmic coincidence which one usually encounters in scalar field DE models and in the concordant $\Lambda \mathrm{CDM}$ model. One resolution of course comes from the consideration of plausible contact interaction(s) between a scalar field induced DE component and the matter field(s) [1,3,27-47], which the scalar-tensor formulations naturally lead to, under conformal transformations [48-57]. A DE-matter (DEM) interaction makes the background matter density $\rho^{(m)}(z)$ drifting from its usual (dust-like) evolution with redshift $z$, thereby affecting the drag force on the matter perturbations. The evolution of the matter density contrast $\delta^{(m)}(z):=\delta \rho^{(m)}(z) / \rho^{(m)}(z)$ and the growth factor $f(z)$ of the matter perturbations are therefore not similar to those in the non-interacting models, in which the field perturbations decay out in the sub-horizon regime, while oscillating about a vanishing mean value. Actually, the decaying nature persists in the interacting scenarios as well, however, with the oscillations about a value proportional to the amount of the interaction, measured by the strength of the scalar field and matter coupling. As such, the field perturbations contribute to the velocity divergences of the matter, affecting in turn the evolution of $\delta^{(m)}(z)[3,58]$. Strikingly enough, a DEM interaction can make the growth factor $f(z)$ acquiring a value $>1$ at large $z$, which necessitates the modifications of the commonly known $f(z)$ parametrizations in the literature [59-64], such as the well-known parametrization $f(z)=\left[\Omega^{(m)}(z)\right]^{\gamma(z)}$, where $\Omega^{(m)}(z)$ is the matter density parameter and $\gamma(z)$ is the so-called growth index [60-72]. Our objective in this paper is to attempt such a modification and demonstrate its utilization in constraining a DEM scenario emerging from a typical scalar-tensor equivalent 'geometric' alternative of GR, viz. the metric-scalartorsion (MST) cosmological theory, formulated recently by one of us (SS) [73-75], on the basis of certain considerations drawn from robust argumentations that have been prevailing for a long time [76-80].

MST essentially forms a class of modified (or 'alternative') gravity theories that contemplates on the appropriate gravitational coupling(s) with scalar field(s) in the RiemannCartan $\left(U_{4}\right)$ space-time geometry, endowed with curvature as well as torsion. The latter being an inherent aspect of a general metric-compatible affine connection is considered as the entity that naturally extends the geometric principles of GR, not only from a classical viewpoint, but also from the perspective of a plausible low energy manifestation of a fundamental (quantum gravitational) theory (see the hefty literature on the vast course of development of the torsion gravity theories in various contexts, the physical implications, and observable effects of torsion thus anticipated, as well as searched extensively over several decades [80-150]) . Nevertheless, conventional $U_{4}$ theories (of Einstein-Cartan type) are faced with a stringent uniqueness problem while taking the minimal couplings with scalar fields into consideration [76-80]. Such couplings are simply not conducive to any unambiguous assertion of equivalent Lagrangians upon eliminating boundary terms in the usual manner. The obvious way out is the consideration of explicit non-minimal (or contact) couplings of the scalar field(s) with, most appropriately, the entire $U_{4}$ Lagrangian given by the $U_{4}$ curvature scalar $\widetilde{R}[73]$. For any particular non-minimal coupling of a scalar field $\phi$ with $\widetilde{R}$, the resulting (MST) action turns out to be equivalent to the scalartensor action, as the trace mode of torsion, $\mathcal{T}_{\mu}$, gets sourced by the field $\phi$, by virtue of the corresponding (auxiliary) equation of motion. On the other hand, torsion's axial (or, pseudo-trace) mode $\mathcal{A}_{\mu}$ can lead to an effective potential, for, e.g., a mass term $m^{2} \phi^{2}$ (with $m=$ constant) in that scalar-tensor equivalent action, upon implementing a norm-fixing constraint $\left(\mathcal{A}_{\mu} \mathcal{A}^{\mu}=\right.$ constant) as in the Einstein-aether theories [151-153], or incorporating a $\phi$-coupled higher order term $\left(\mathcal{A}_{\mu} \mathcal{A}^{\mu}\right)^{2}$ [73]. Such a mass term is shown to play a crucial role in giving rise to a viable cosmological scenario marked by an $\phi$-induced DE component with a weak enough dynamical evolution amounting to cosmological parametric estimations well within the corresponding observational error limits for $\Lambda \mathrm{CDM}$. This also corroborates to the local gravitational bounds on the effective Brans-Dicke (BD) parameter $\mathfrak{w}$, which turns out to be linear in the inverse of the MST coupling parameter $\beta$ [73]. 
Particularly intriguing is the MST cosmological scenario that emerges under a conformal transformation from the Jordan frame to the Einstein frame, in which the effective DE component interacts with the cosmological matter (a priori in the form of $d u s t$ ). Nevertheless, the crude estimate of $\beta$ (or of the parameter $s=2 \beta$ that appears in the exact solution of the Friedmann equations), obtained under the demand of a small deviation from the background $\Lambda C D M$ evolution [73], requires a robust reconciliation at the perturbative level. On the other hand, the methodology adopted here can in principle apply to any scalar-tensor cosmological scenario, once we resort to the dynamics in the Einstein frame.

Now, the methodology of our analysis purports to fulfill our objective mentioned above. Specifically, we take the following course and organize this paper accordingly: in Section 2, we review the basic tenets of MST cosmology in the standard FRW framework, and, in particular, the exact solution of the cosmological equations in the Einstein frame that describes a typical interacting DE evolution. Then, in the initial part of Section 3, we obtain the differential equations for $\delta^{(m)}(z)$ and $f(z)$, and get their evolution profiles by numerically solving those equations for certain fiducial settings of the parameters $s=2 \beta$ and $\left.\Omega_{0}^{(m)} \equiv \Omega^{(m)}\right|_{z=0}$. Thereafter, in Section 3.1, we resort to a suitable growth factor parametrization, demanding that an appropriate expansion of the growth index $\gamma(z)$ about the present epoch $(z=0)$ should adhere to the observational constraints on the growth history predictions at least up to $z \simeq 1$ or so. Next, in Section 3.2, we attain the pre-requisites for the growth data fitting with the quantity $f \sigma^{(8)}(z)$, where $f(z)$ is as given by its chosen parametrization, and $\sigma^{(8)}(z)$ is the root-mean-square amplitude of matter perturbations within a sphere of radius $8 \mathrm{Mph}^{-1}$. Finally, in Section 4 , we estimate the requisite parameters $s, \Omega_{0}^{(m)}$ and $\left.\sigma_{0}^{(8)} \equiv \sigma^{(8)}\right|_{z=0}$, and hence constrain the model by fitting $f \sigma^{(8)}(z)$ with a refined sub-sample of the redshift-space-distortion (RSD) data, and its combination with the observational Hubble data [154]. In Section 5, we conclude with a summary of the work and an account on some open issues.

Conventions and Notations: We use metric signature $(-,+,+,+)$ and natural units (with the speed of light $c=1$ ), and denote the metric determinant by $g$, the Planck length parameter by $\kappa=\sqrt{8 \pi G_{N}}$ (where $G_{N}$ is the Newton's gravitational constant) and the values of parameters or functions at the present epoch by an affixed subscript ' 0 '.

\section{MST Cosmology in the Einstein Frame and the Emergent DEM Interacting Scenario}

As mentioned above, an intriguing scenario of an effective DEM interaction emerges from a typical scalar-tensor equivalent MG formulation, viz. the one involving a nonminimal metric-scalar-torsion (MST) coupling, in the Einstein frame [73]. Let us first review briefly the main aspects of such a formalism, and the emergent cosmological scenario in the standard FRW framework.

Torsion, by definition, is a third rank tensor $T_{\mu \nu}^{\alpha}$ which is anti-symmetric in two of its indies ( $\mu$ and $v$ ) because of being the resultant of the anti-symmetrization of a general asymmetric affine connection $\left(\widetilde{\Gamma}_{\mu \nu}^{\alpha} \neq \widetilde{\Gamma}_{\nu \mu}^{\alpha}\right)$ that characterizes the four-dimensional Riemann-Cartan (or $U_{4}$ ) space-time geometry. The latter, however, demands the metriccompatibility, viz. the condition $\widetilde{\nabla}_{\alpha} g_{\mu \nu}=0$, where $\widetilde{\nabla}_{\alpha}$ is the $U_{4}$ covariant derivative defined in terms of the corresponding connection $\widetilde{\Gamma}_{\mu \nu}^{\alpha}$. Such a condition leads to a lot of simplification in the expression for the $U_{4}$ curvature scalar equivalent, $\widetilde{R}$, which is usually considered as the free $U_{4}$ Lagrangian analogously with the free gravitational Lagrangian in GR, viz. the Riemannian (or $R_{4}$ ) curvature scalar $R$. Specifically, $\widetilde{R}$ gets reduced to a form given by $R$, plus four torsion-dependent terms proportional to the norms of irreducible modes, viz. the trace vector $\mathcal{T}_{\mu} \equiv T_{\mu \alpha}^{\alpha}$, the pseudo-trace vector $\mathcal{A}^{\mu}:=\epsilon^{\alpha \beta \gamma \mu} T_{\alpha \beta \gamma}$ and the (pseudo)tracefree tensor $\mathcal{Q}_{\mu \nu}^{\alpha}:=T_{\mu \nu}^{\alpha}+\frac{1}{3}\left(\delta_{\mu}^{\alpha} \mathcal{T}_{v}-\delta_{v}^{\alpha} \mathcal{T}_{\mu}\right)-\frac{1}{6} \epsilon_{\mu \nu \sigma}^{\alpha} \mathcal{A}^{\alpha}$, as well as the covariant divergence of $\mathcal{T}_{\mu}$ [80]. In the absence of sources (or the generators of the so-called canonical spin density), all the torsion terms drop out, and hence the $U_{4}$ theory effectively reduces to GR. The situation remains the same for minimal couplings with scalar fields as well. However, such couplings are themselves problematic, when it comes to assigning 
the effective Lagrangian uniquely upon eliminating the boundary terms [76-80]. An easy cure is to resort to distinct non-minimal couplings of a given scalar field $\phi$, in general, with each of the constituent terms in $\widetilde{R}$ [80]. However, this implies the involvement of more than one arbitrary coupling parameters, which may affect the predictability and elegance of the theory. Hence, it is much reasonable to consider a non-minimal $\phi$-coupling with the entire $\widetilde{R}$, so that there is a unique (MST) coupling parameter (to be denoted by $\beta$, say) [73].

Eliminating boundary terms, we obtain the auxiliary equation (or the constraint) $\mathcal{T}_{\mu}=3 \phi^{-1} \partial_{\mu} \phi$, which implies that the (presumably primordial, and a priori massless) scalar field $\phi$ acts as a source of the trace mode of torsion. Considering further a mass term $m^{2} \phi^{2}$ induced by torsion's axial mode $\mathcal{A}_{\mu}$, via one of the possible ways mentioned above (in the Introduction), we get the following effective MST action ignoring, of course, any external source for the tensorial mode $\mathcal{Q}^{\alpha}{ }_{\mu \nu}$, which therefore vanishes identically (note that this is particularly relevant to what we intend to study here, viz. a homogeneous and isotropic cosmological evolution in the presence of torsion, which is plausible only when the latter's modes are severely constrained, and one such constraint is $\mathcal{Q}^{\alpha}{ }_{\mu \nu}=0$ [97]):

$$
\mathcal{S}=\int d^{4} x \sqrt{-g}\left[\frac{\beta \phi^{2}}{2} R-\frac{1-6 \beta}{2} g^{\mu v} \partial_{\mu} \phi \partial_{\nu} \phi-\frac{1}{2} m^{2} \phi^{2}+\mathcal{L}^{(m)}\left(g_{\mu v},\{\psi\}\right)\right] .
$$

This is nothing but the scalar-tensor action in the presence of minimally coupled matter fields $(\{\psi\})$ described by the Lagrangian $\mathcal{L}^{(m)}$, in the Jordan frame [73].

Under a conformal transformation $g_{\mu v} \rightarrow \widehat{g}_{\mu v}=\left(\phi / \phi_{0}\right)^{2} g_{\mu v}$ and field redefinition $\varphi:=\phi_{0} \ln \left(\phi / \phi_{0}\right)$, with $\phi_{0}=(\kappa \sqrt{\beta})^{-1}$ - the value of $\phi$ at the present epoch $t=t_{0}$, one obtains the Einstein frame MST action

$$
\widehat{\mathcal{S}}=\int d^{4} x \sqrt{-\widehat{g}}\left[\frac{\widehat{R}}{2 \kappa^{2}}-\frac{1}{2} \widehat{g}^{\mu v} \partial_{\mu} \varphi \partial_{\nu} \varphi-\Lambda e^{-2 \varphi / \phi_{0}}+\widehat{\mathcal{L}}^{(m)}\left(\widehat{g}_{\mu \nu}, \varphi,\{\psi\}\right)\right],
$$

where $\widehat{R}$ is the corresponding (Ricci) curvature scalar, and $\kappa=\sqrt{8 \pi G_{N}}$ denotes the gravitational coupling factor. Note that this can be actually be retrieved from the relationship $\kappa=\left(\phi / \phi_{0}\right) \kappa_{\text {eff }}(\phi)$, where $\kappa_{\text {eff }}(\phi)=(\phi \sqrt{\beta})^{-1}$ is the effective (running) gravitational coupling one has in the Jordan frame. The parameter $\Lambda=\frac{1}{2} m^{2} \phi_{0}^{2}$, which amounts to the effective field potential at $t=t_{0}$, and

$$
\widehat{\mathcal{L}}^{(m)}\left(\widehat{g}_{\mu v}, \varphi,\{\psi\}\right)=e^{-4 \varphi / \phi_{0}} \mathcal{L}^{(m)}\left(g_{\mu v},\{\psi\}\right)
$$

is the transformed matter Lagrangian, which depends on the field $\varphi$ both explicitly as well as implicitly (since $g_{\mu v}=g_{\mu v}\left(\widehat{g}_{\mu v}, \varphi\right)$ ). It is in fact this $\varphi$-dependence which leads to the DEM interaction in the standard cosmological setup, as we shall see below. Note also that, by definition, $\left.\varphi\right|_{t=t_{0}}=0$.

Dropping the hats $(\Im)$, we express the gravitational field equation and the individual matter and field (non-)conservation relations in the Einstein frame as follows:

$$
\begin{aligned}
& R_{\mu \nu}-\frac{1}{2} g_{\mu \nu} R=\kappa^{2}\left[T_{\mu \nu}^{(m)}+T_{\mu \nu}^{(\varphi)}\right] \\
& \nabla_{\alpha}\left(g^{\alpha \nu} T_{\mu \nu}^{(m)}\right)=-\nabla_{\alpha}\left(g^{\alpha \nu} T_{\mu \nu}^{(\varphi)}\right)=-\frac{T^{(m)} \partial_{\mu} \varphi}{\phi_{0}},
\end{aligned}
$$

where $T_{\mu v}^{(m)}$ and $T_{\mu \nu}^{(\varphi)}$ are the respective energy-momentum tensors for matter and scalar field, and $T^{(m)} \equiv g^{\mu \nu} T_{\mu \nu}^{(m)}$ denotes the trace of $T_{\mu \nu}^{(m)}$.

Considering the matter to be a priori in the form of a pressure-less fluid (viz. 'dust'), we have in the standard spatially flat FRW framework, $T_{v}^{\mu(m)}=\operatorname{diag}\left[-\rho^{(m)}, 0,0,0\right]$, so that $-T^{(m)}=\rho^{(m)}$ is just the matter density, which is purely a function of the cosmic time $t$. Because of the interaction (5), the matter density ceases to have its usual dust-like 
evolution (i.e., $\rho^{(m)}(t) \not a^{-3}(t)$, where $a(t)$ is the FRW scale factor). Nevertheless, the above Equations (4) and (5) are shown to be solvable in an exact analytic way, for the configuration

$$
\varphi(t)=s \phi_{0} \ln [a(t)], \quad \rho^{(m)}(t) \propto a^{-(3+s)}(t),
$$

provided one sets the constant parameter $s=2 \beta$ [73]. Consequently, the matter density parameter $\Omega^{(m)}(a)$ is expressed as

$$
\Omega^{(m)}(a):=\frac{\rho^{(m)}(a)}{\rho(a)}=\frac{(3-s) \Omega_{0}^{(m)} a^{-(3-s)}}{3 \Omega_{0}^{(m)}\left[a^{-(3-s)}-1\right]+3-s},
$$

where $\rho(a)$ is the total (or critical) density of the universe, and $\Omega_{0}^{(m)}$ is the value of $\Omega^{(m)}$ at the present epoch $\left(t=t_{0}\right.$, whence $\left.a=1\right)$. Using the Friedmann and Raychaudhuri equations, we can then express the Hubble parameter and total EoS parameter of the system, respectively, as

$$
\begin{gathered}
H(a):=\frac{\dot{a}}{a}=H_{0}\left(1-\frac{s}{3}\right)^{-1 / 2}\left[\Omega_{0}^{(m)} a^{-(3+s)}+\left(1-\frac{s}{3}-\Omega_{0}^{(m)}\right) a^{-2 s}\right]^{1 / 2}, \\
\mathrm{w}(a):=\frac{p(a)}{\rho(a)}=-1+\Omega^{(m)}(a)+\frac{2 s}{3},
\end{gathered}
$$

where $H_{0}=H(a=1)$ is the Hubble constant, and $p(a)$ denotes the total pressure. Note that, in the limit $s \rightarrow 0$, the above equations reduce to the corresponding ones for $\Lambda \mathrm{CDM}$. Therefore, one can directly estimate the extent to which the MST cosmological scenario can deviate from $\Lambda \mathrm{CDM}$, by demanding that such a deviation should not breach the corresponding $68 \%$ parametric margins for $\Lambda \mathrm{CDM}$. This would in turn provide an estimation of the parameter $s$, which has actually been carried out in [73], using the Planck 2015 and the WMAP 9 year results. The upper bound on $s$, thus obtained, is of the order of $10^{-2}$. Nevertheless, a rather robust reconciliation is required from an independent analysis, for instance, using the RSD and $H(z)$ observations, which we endeavor to do in this paper.

\section{Growth of Matter Density Perturbations}

In this section, we discuss the evolution of linear matter density perturbations in the deep sub-horizon regime for the aforementioned Einstein frame background MST cosmological scenario. The perturbations can be studied in the well-defined conformal Newtonian gauge. The metric in this gauge is given as [3]

$$
d s^{2}=e^{2 N}\left[-(1-2 \Phi) \mathcal{H}^{-2} d N^{2}+(1+2 \Phi) \delta_{i j} d x^{i} d x^{j}\right],
$$

where $N:=\ln a(t)$ is the number of e-foldings, $\mathcal{H}$ is the conformal Hubble parameter, and $\Phi$ is the Bardeen potential. Note that we have taken the same potential $\Phi$ in both temporal and spatial part of the metric under the assumption of a vanishing anisotropic stress.

The evolution of the matter density contrast $\delta^{(m)}$ depends on the divergence or convergence of the peculiar velocity $\overrightarrow{\boldsymbol{v}}^{(m)}$ via the perturbed continuity equation

$$
\frac{d \delta^{(m)}}{d N}=-\theta^{(m)}, \quad \text { where } \theta^{(m)}:=\nabla \cdot \overrightarrow{\boldsymbol{v}}^{(m)} .
$$

On the other hand, the Euler equation for matter perturbations is given by

$$
\frac{d \theta^{(m)}}{d N}=-\left[\frac{\theta^{(m)}}{2}\left(1-3 w-\kappa \sqrt{2 s} \frac{d \varphi}{d N}\right)+\widehat{\lambda}^{-2}\left(\Phi+\kappa \sqrt{\frac{s}{2}} \delta \varphi\right)\right]
$$


where $\widehat{\lambda} \equiv \mathcal{H} / k$ (with $k$ being the comoving wavenumber), and

$$
\Phi \simeq \frac{3}{2} \widehat{\lambda}^{2} \Omega^{(m)} \delta^{(m)}, \quad \delta \varphi \simeq 3 \widehat{\lambda}^{2} \sqrt{\frac{s}{2}} \Omega^{(m)} \delta^{(m)},
$$

considering the latter to be the mean value of the field perturbation, which shows a damped oscillatory behavior in the sub-horizon regime.

$\Phi$ and $\delta \varphi$ both being proportional to $\widehat{\lambda}^{2}$, become negligible in the deep sub-horizon limit $\left(\widehat{\lambda}^{2} \ll 1\right)$. However, their contribution may not be negligible in the evolution of $\theta^{(m)}(N)$ because of the $\widehat{\lambda}^{-2}$ pre-factor in the second term of Equation (12). As a consequence, the DE perturbation $\delta \varphi$, which itself is negligible in the sub-horizon regime (despite being scale-dependent), may, by virtue of its coupling with matter, lead to a significant effect on the growth of matter density perturbations.

Equations (9), (11) and (12) yield the second-order differential equation

$$
\frac{d \delta^{(m)}}{d N}+\left[2(1-s)-\frac{3 \Omega^{(m)}}{2}\right] \frac{d \delta^{(m)}}{d N}=\frac{3(1+s)}{2} \Omega^{(m)} \delta^{(m)},
$$

which can be reduced to the following first-order differential equation:

$$
\frac{d f}{d N}+f^{2}+\left[2(1-s)-\frac{3 \Omega^{(m)}}{2}\right] f=\frac{3(1+s)}{2} \Omega^{(m)},
$$

by defining the so-called growth factor $f(N):=d\left[\ln \delta^{(m)}\right] / d N$ [155-159]. Due to the prefactor $(1+s)$ in the r.h.s. of Equation (15), the function $f(N)$ can cross the unity barrier at high redshifts (whence $\Omega^{(m)} \rightarrow 1$ ). This is illustrated in Figure 1a, where we have plotted $f(z)$ for a fixed $\Omega_{0}^{(m)}=0.3$ and certain fiducial values of $s$, including $s=0$ (the $\Lambda \mathrm{CDM}$ case). Figure $1 \mathrm{~b}$, on the other hand, depicts the evolution of $\delta^{(m)}(z)$, which tends to increase with $s$ for a fixed $\Omega_{0}^{(m)}=0.3$.

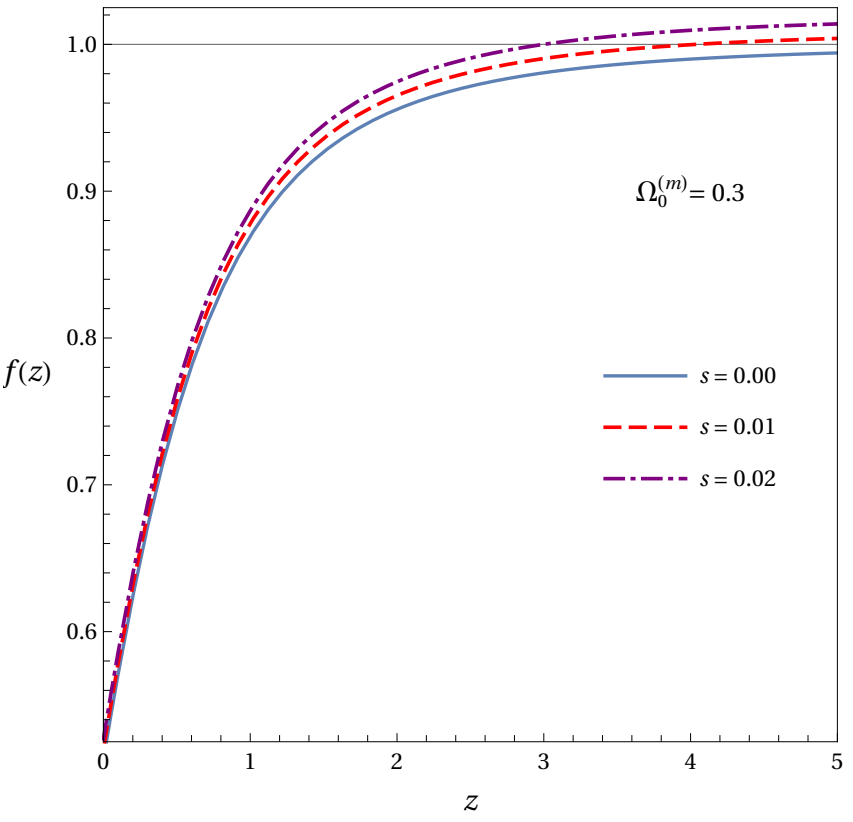

(a)

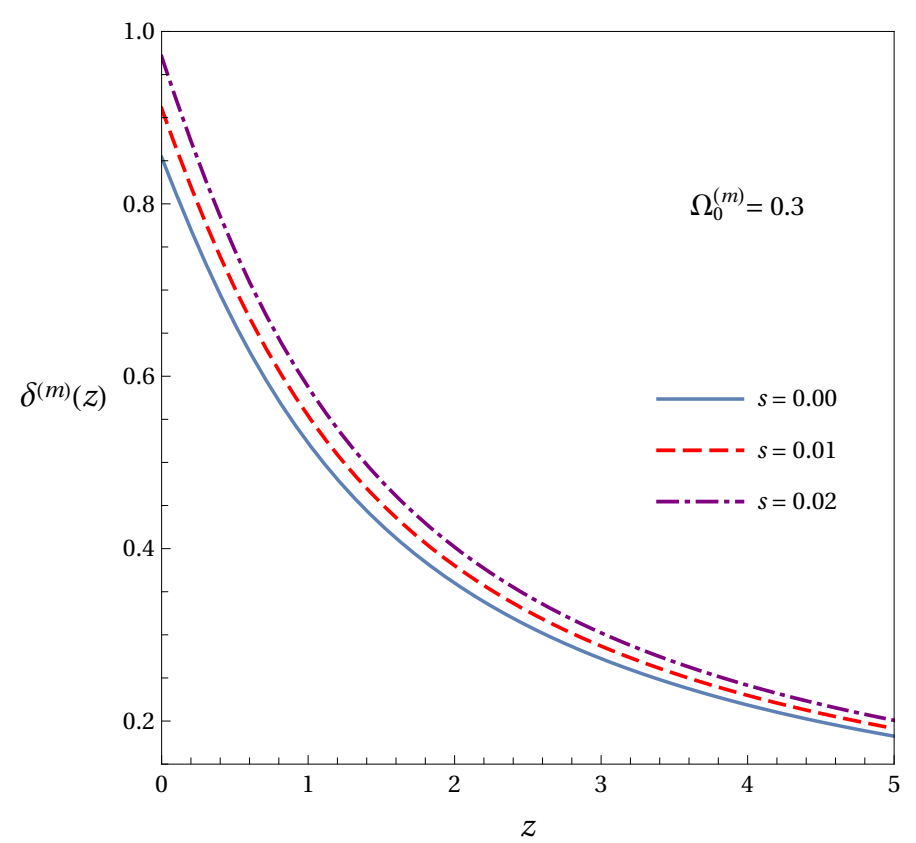

(b)

Figure 1. Functional variations of (a) the growth factor $f(z)$ and $(\mathbf{b})$ the matter density contrast $\delta^{(m)}(z)$, in the redshift range $z \in[0,5]$, for the fiducial parametric settings $\Omega_{0}^{(m)}=0.3$ (fixed) and $s=0,0.01$, and 0.02 . 


\subsection{Growth Factor Parametrization}

As mentioned earlier, following the well-known prescription of $[59,60]$, we may consider parametrizing the growth factor $f(z)$ as $\left[\Omega^{(m)}(z)\right]^{\gamma(z)}$. However, such a parametrization does not explain the crossing of $f(z)$ from $<1$ to $>1$ at large-redshifts, as illustrated in Figure 1a. More precisely, this parametrization $f(z)$ is always restricted within the range $[0,1]$ at all redshifts which in our case is not true. Thus, to alleviate this limitation, we propose the ansatz:

$$
f(z)=(1+s)\left[\Omega^{(m)}(z)\right]^{\gamma(z)},
$$

which evidently implies $f(z)$ approaching $1+s$ at large redshifts (whence $\Omega^{(m)} \rightarrow 1$ ). Now, to determine the growth rate of matter perturbations from Equation (16), it is necessary to find a suitable functional form of $\gamma(z)$. In particular, choosing to express the growth index as a function of the scale factor $a$, we in this paper resort to the following truncated form of its Taylor expansion about $a=1$ (which corresponds to the present epoch):

$$
\gamma(a)=\gamma_{0}+\gamma_{1}(1-a), \quad \text { with } \gamma_{0}, \gamma_{1}:=\text { constants },
$$

as in [62,70]. Note that this parametrization is valid at least up to a redshift $z \simeq 1$ and is therefore suitable for the analysis using the RSD observational dataset [160-162], as most of the data points in that the set lies within $z=1$. In fact, it is rather convenient for us to re-write Equation (17) as

$$
\gamma(N)=\gamma_{0}+\gamma_{1}\left(1-e^{N}\right),
$$

where

$$
\begin{array}{r}
\gamma_{0}=\left.\gamma(N)\right|_{N=0}=\frac{1}{\ln \Omega_{0}^{(m)}} \ln \left(\frac{f_{0}}{1+s}\right) \quad\left[\text { with } f_{0}=\left.f\right|_{N=0}\right], \\
\gamma_{1}=\left.\frac{d \gamma(N)}{d N}\right|_{N=0}=\frac{1}{\ln \Omega_{0}^{(m)}}\left[\gamma_{0}\left(s-3+3 \Omega_{0}^{(m)}\right)+(1+s)\left(\Omega_{0}^{(m)}\right)^{\gamma_{0}}\right. \\
\left.+2(1-s)-\frac{3}{2}\left(\Omega_{0}^{(m)}+\left(\Omega_{0}^{(m)}\right)^{1-\gamma_{0}}\right)\right],
\end{array}
$$

by Equations (15) and (16).

For the $\Lambda$ CDM case $(s=0)$, assuming $\Omega_{0}^{(m)}=0.3$, one gets $\gamma_{0} \simeq 0.555$ and $\gamma_{1} \simeq$ -0.016 . Moreover, the signature of $\gamma_{1}$ can discriminate between various DE models and modified gravity theories. For instance, the minimal level Dvali-Gabadadze-Porrati (DGP) model predicts $\left(0.035<\gamma_{1}<0.042\right)$, which is in sharp contrast to the GR predictions [62].

\subsection{Numerical Fitting of the Growth Index}

Let us now focus on determining the parametric set $p(\theta)=\left\{s, \Omega_{0}^{(m)}, \sigma_{0}^{(8)}, \gamma_{0}, \gamma_{1}\right\}$. While the form of the parameter $\gamma_{1}$ is already obtained in terms of $s, \Omega_{0}^{(m)}$, and $\gamma_{0}$, we require to assert the form of $\gamma_{0}$ in the first place. However, as we see from Equation (19), $\gamma_{0}$ depends on $s$ and $\Omega_{0}^{(m)}$ as well. Hence, we resort to solving numerically the differential Equation (15), by taking $s \in[0,0.1]$ and $\Omega_{0}^{(m)} \in[0.2,0.4]$ (which are, of course, a fairly wide range of values), and for a step-size of 0.01. Using Equation (19), thereafter, we obtain the following fit:

$$
\gamma_{0} \simeq \frac{0.547}{\left[\Omega_{0}^{(m)}\right]^{0.012}}-1.118 s \Omega_{0}^{(m)} .
$$

In order to verify the validity of this fitting, let us take the $\Omega_{0}^{(m)}=0.3$, say, and the limit $s \rightarrow 0$. Equation (21) then gives $\gamma_{0} \simeq 0.555$ which is precisely what we had estimated theoretically, for the $\Lambda \mathrm{CDM}$ case, in the last subsection, by using Equations (15) and (19). The goodness of the fit is illustrated in Figure 2a,b, in which we have plotted the fractional error in the fitting, viz. $E_{f}(z):=\left[f_{F}(z)-f(z)\right] / f(z)$ with $z \in[0,2.5]$, for a fixed $\Omega_{0}^{(m)}$ 
and a range of fiducial values of $s$, and for a fixed $s$ and a range of fiducial values of $\Omega_{0}^{(m)}$, respectively. In both the cases, the error turns out to be $\simeq 0.2 \%$ at $z \simeq 1$, indicating a fair amount of the accuracy of the fit.

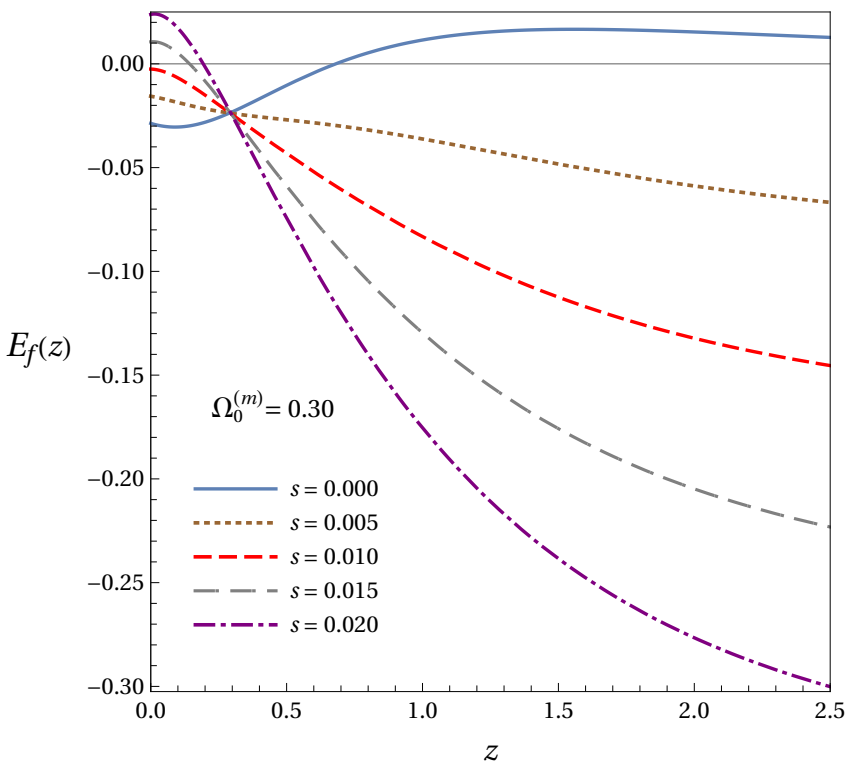

(a)

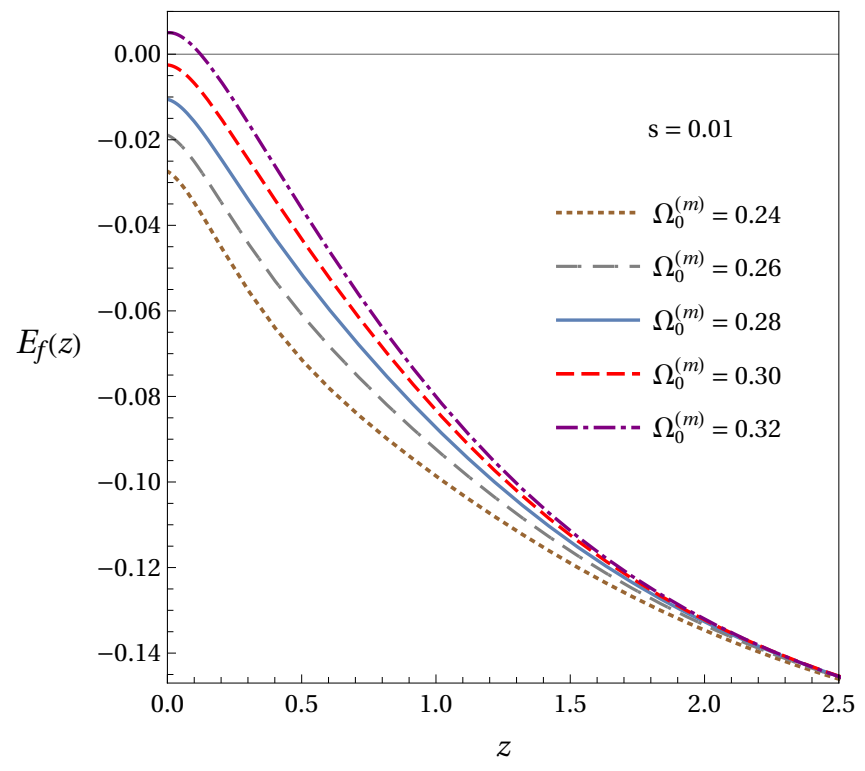

(b)

Figure 2. Functional variations of the growth factor fitting error, $E_{f}(z)$, in the redshift range $z \in[0,2.5]$, for the fiducial parametric settings (a) $\Omega_{0}^{(m)}=0.3$ (fixed) and variable $s \in[0,0.02] ;(\mathbf{b}) s=0.01$ (fixed) and variable $\Omega_{0}^{(m)} \in[0.24,0.32]$.

\section{Parametric Estimations from RSD and Hubble Observations}

After formulating $\gamma_{0}$ and $\gamma_{1}$ in terms of $s$ and $\Omega_{0}^{(m)}$, we are left with only three parameters $s, \Omega_{0}^{(m)}$, and $\sigma_{0}^{(8)}$ in hand. Thus, in order to estimate them from the observations, use the $f \sigma^{(8)}(z)$ observations from various galaxy data surveys [160-170], we will now proceed to perform the statistical analysis, and in particular the Markov-Chain-MonteCarlo (MCMC) simulation to estimate our model parameters. Theoretically, $\left(f \sigma^{(8)}\right)_{t h}(z)$ can be written as [171-176]

$$
\left(f \sigma^{(8)}\right)_{t h}(z)=f(z) \sigma_{0}^{(8)} \frac{\delta^{(m)}(z)}{\delta_{0}^{(m)}}, \quad \text { where } \quad \sigma_{0}^{(8)}=\left.\sigma^{(8)}\right|_{z=z_{0}} .
$$

This can be explicitly written as

$$
\left(f \sigma^{(8)}\right)_{t h}(N)=\sigma_{0}^{(8)}(1+s)\left(\Omega^{(m)}\right)^{\gamma(N)} \exp \left[(1+s) \int_{0}^{N}\left(\Omega^{(m)}\right)^{\gamma(N)} d N\right],
$$

where we have used Equation (16). Since our parameter $s$ is presumably positive definite and small, it is convenient for us to write $s=|\widetilde{s}|$, where $\widetilde{s}$ can take both positive and negative values.

In order to perform the standard $\chi^{2}$ minimization, we use the growth data observations: $A_{o b s} \equiv\left(f \sigma^{(8)}\right)_{o b s}$ along with the theoretical predicted values: $A_{t h} \equiv\left(f \sigma^{(8)}\right)_{t h}$ in the standard definition of the $\chi^{2}$ function

$$
\chi^{2}:=V^{m} C_{m n}^{-1} V^{n},
$$

where $V:=A_{o b s}-A_{t h}$ and $C_{m n}^{-1}$ is the inverse of the covariance matrix between three WiggleZ data points [176]. As we have already shown in Figure 2 that the parametric form (18) tends to diverge in case of interacting DE from its numerical solution (16) at high redshifts, we therefore restrict ourselves for the observations up to $z=1$ for the datasets: 
GOLD-2017 [173] and $H(z)$ data set [154]. In addition, we set the range of priors as follows: (i) $-1 \leq \widetilde{s} \leq 1$, (ii) $0.1 \leq \Omega_{0}^{(m)} \leq 0.6$, (iii) $0.5 \leq \sigma_{0}^{(8)} \leq 1.2$ and (iv) $0.4 \leq h \leq 0.9$, where $h:=H_{0} /\left[100 \mathrm{Km} \mathrm{s}^{-1} \mathrm{Mpc}^{-1}\right]$. The obtained contour plots between parameters up to a $3 \sigma$ level are shown in Figure 3a,b.

The estimations are shown in Table 1 in which one can see that the best-fit of $\widetilde{s}$ for both sets of data (GOLD and GOLD $+H(z)$ ) is insignificant (as expected, since observations mostly prefer the $\Lambda \mathrm{CDM}$ model), but even then, within $1 \sigma$ limits, its domain can reach up to a significantly large value i.e., $\mathcal{O}\left(10^{-2}\right)$, which shows a reasonably large deviation from the $\Lambda \mathrm{CDM}$ model. This indicates, from the low-redshift data, that we can still observe a convincing amount of DEM interaction even at the $1 \sigma$ level.

Table 1. Best fit values with $1 \sigma$ confidence limits of parameters $\Omega_{0}^{(m)}, \sigma_{0}^{(8)}, h$, and $\widetilde{s}$, together with their corresponding $\chi^{2} /$ dof, for the GOLD and GOLD $+H(z)$ dataset.

\begin{tabular}{|c|c|c|c|c|c|}
\hline \multirow{2}{*}{ Observational Datasets } & \multicolumn{4}{|c|}{$\begin{array}{l}\text { Parametric Estimations } \\
\text { (Best Fit \& 68\% Limits) }\end{array}$} & \multirow{2}{*}{$\chi^{2} / d o f$} \\
\hline & $\Omega_{0}^{(m)}$ & $\sigma_{0}^{(8)}$ & $h$ & $\widetilde{s}$ & \\
\hline 1. GOLD & $0.2610_{-0.0451}^{+0.0487}$ & $0.7460_{-0.0466}^{+0.0476}$ & - & $0.0319_{-0.0702}^{+0.0683}$ & 0.8390 \\
\hline 2. GOLD $+H(z)$ & $0.2753_{-0.0387}^{+0.0393}$ & $0.7417_{-0.0402}^{+0.0393}$ & $0.6804_{-0.0183}^{+0.0189}$ & $0.0308_{-0.0573}^{+0.0573}$ & 0.6125 \\
\hline
\end{tabular}

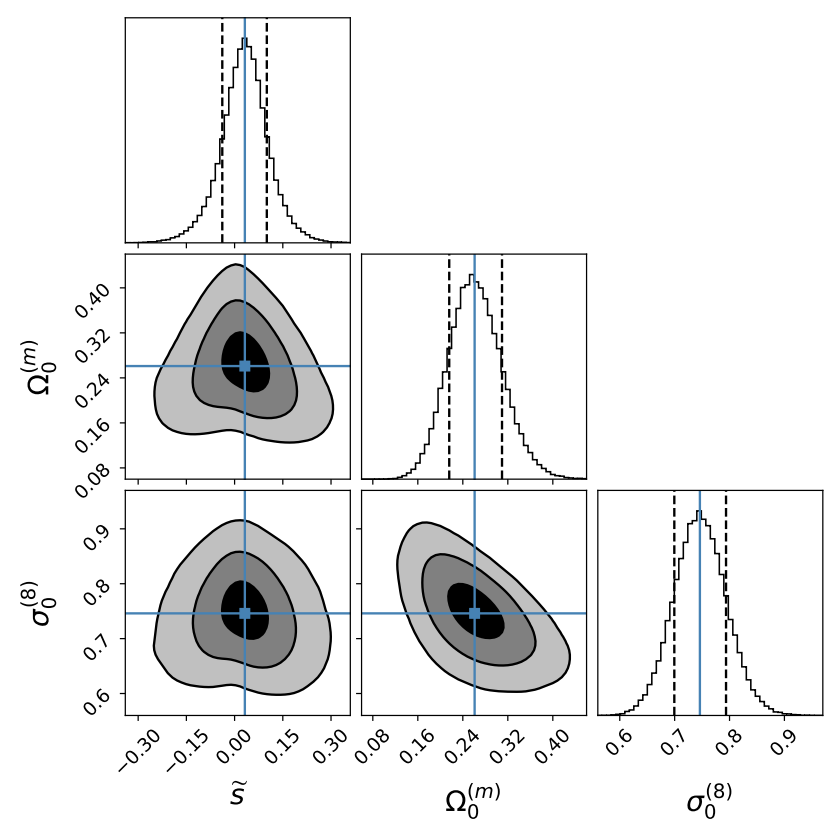

(a)

Figure 3. Cont. 


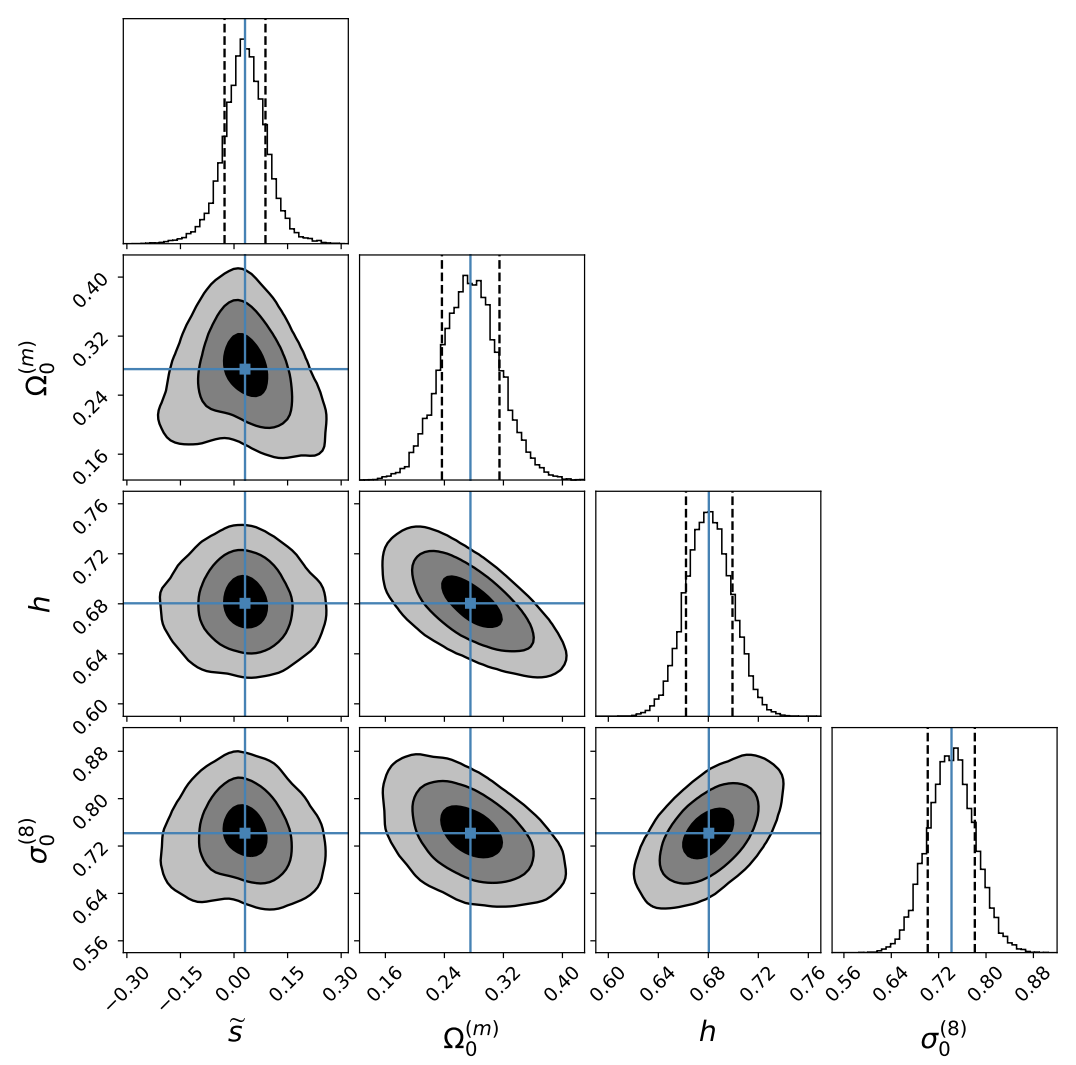

(b)

Figure 3. 2D posterior distribution ( $1 \sigma-3 \sigma$ contour levels) for (a) the Gold dataset, and (b) its combination with the Hubble dataset. The solid blue lines denote the respective best-fits and dashed lines correspond to the respective $1 \sigma$ levels.

\section{Conclusions}

We have formulated the growth of linear matter density perturbations in a parametric form for a DE model which stems from a modified gravity approach that consists of metric and torsion as two basic entities of the space-time geometry. In the formalism, we have briefly demonstrated that a non-minimal coupling of metric and torsion with a scalar field can give rise to a scalar-tensor action of DE in the Jordan frame which upon conformal transformation to the Einstein frame naturally makes a scalar field non-minimally coupled with the matter sector. Due to this coupling, matter and scalar field exchange their energies between each other, which stops their individual energy densities from being self-conserved. The latter, thus, has a direct influence on the underline matter density contrast and its evolution, which we have explored in this work.

We have demonstrated that, in the perturbed FRW space-time, the scalar field and matter coupling enhances the growth of matter density perturbations in the sub-horizon regime, allowing it to cross the upper barrier of unity at large redshifts. Since this effect is unique in the interacting DEM scenarios, it requires a slight modification in the standard parametric ansatz of a growth factor. With suitable modification, we propose a slightly different growth factor ansatz to make the parametric formulation compatible with the theoretical predictions. In addition, in view of the time evolving growth index, which is even encountered for the $\Lambda \mathrm{CDM}$ model, we have chosen an appropriate functional form i.e., first order Taylor expansion about a present-day value of the scale factor $a(t)$. This simple but well defining form of the growth index indeed illustrates the parametric formulation of the growth factor close to its actual evolution at least up to $z \simeq 1$. Since the present-day value of the growth index depends itself on the background model parameters, therefore, in order to choose its explicit function form, we have numerically obtained its 
fitting formula in terms of coupling as well as energy density parameter which we have shown to be a well approximation for a wide range of parameters.

As to the parametric estimations, we have constrained parameters $\widetilde{s}, \Omega_{0}^{(m)}$, and $\sigma_{0}^{(8)}$ by using the RSD as well as its combination with the Hubble data. We have found that, for the GOLD dataset, the $\widetilde{s}$ and hence the $s$ parameter can lead to a mildly large deviation from the $\Lambda$ CDM model up to $1 \sigma$, which is comparatively smaller for the combined dataset, as expected. The consistency in our estimations with the theoretical predictions confirms the validity of our fitting function. However, to explain growth history for redshifts $>1$, the above parametrization still requires further modifications to deal with various DE models as well as modified gravity theories, which we will shall endeavor to report in future.

Acknowledgments: The work of M.K.S. was supported by the Council of Scientific and Industrial Research (CSIR), Government of India.

\section{References}

1. Copeland, E.J.; Sami, M.; Tsujikawa, S. Dynamics of dark energy. Int. J. Mod. Phys. D 2006, 15, 1753-1935. [CrossRef]

2. Frieman, J.A.; Turner, M.S.; Huterer, D. Dark energy and the accelerating universe. Ann. Rev. Astron. Astrophys. 2008, 46, 385-432. [CrossRef]

3. Amendola, L.; Tsujikawa, S. Dark Energy: Theory and Observations; Cambridge University Press: Cambridge, UK, 2010.

4. Wolschin, G. Lectures on Cosmology: Accelerated Expansion of the Universe; Springer: Berlin/Heidelberg, Germany, 2010.

5. Matarrese, S.; Colpi, M.; Gorini, V.; Moschella, U. Dark Matter and Dark Energy: A Challenge for Modern Cosmology; Springer: Dordrecht, The Netherlands, 2011.

6. Hinshaw, G.; Larson, D.; Komatsu, E.; Spergel, D.N.; Bennett, C.; Dunkley, J.; Nolta, M.R.; Halpern, M.; Hill, R.S.; Odegard, N.; et al. Nine-year Wilkinson Microwave Anisotropy Probe (WMAP) Observations: Cosmological Parameter Results. Astrophys. J. Suppl. 2013, 208, 19. [CrossRef]

7. Bennett, C.L.; Larson, D.; Weiland, J.L.; Jarosik, N.; Hinshaw, G.; Odegard, N.; Smith, K.M.; Hill, R.S.; Gold, B.; Halpern, M.; et al. Nine-Year Wilkinson Microwave Anisotropy Probe (WMAP) Observations: Final Maps and Results. Astrophys. J. Suppl. 2013, 208, 20. [CrossRef]

8. Betoule, M.E.; Kessler, R.; Guy, J.; Mosher, J.; Hardin, D.; Biswas, R.; Astier, P.; El-Hage, P.; Konig, M.; Kuhlmann, S.; et al. Improved cosmological constraints from a joint analysis of the SDSS-II and SNLS supernova samples. Astron. Astrophys. 2014, 568, A22. [CrossRef]

9. Ade, P.A.; Aghanim, N.; Arnaud, M.; Ashdown, M.; Aumont, J.; Baccigalupi, C.; Banday, A.J.; Barreiro, R.B.; Bartlett, J.G.; Bartolo, N.; et al. Planck 2015 results, XIII. Cosmological parameters. Astron. Astrophys. 2016, 594, A13.

10. Ade, P.A.; Aghanim, N.; Arnaud, M.; Ashdown, M.; Aumont, J.; Baccigalupi, C.; Banday, A.J.; Barreiro, R.B.; Bartolo, N.; Battaner, E.; et al. Planck 2015 results, XIV. Dark energy and modified gravity. Astron. Astrophys. 2016, 594, A14.

11. Scolnic, D.M.; Jones, D.O.; Rest, A.; Pan, Y.C.; Chornock, R.; Foley, R.J.; Huber, M.E.; Kessler, R.; Narayan, G.; Riess, A.G.; et al. The Complete Light-curve Sample of Spectroscopically Confirmed SNe Ia from Pan-STARRS1 and Cosmological Constraints from the Combined Pantheon Sample. Astrophys. J. 2018, 859, 101. [CrossRef]

12. Planck Collaboration; Aghanim, N.; Akrami, Y.; Ashdown, M.; Aumont, J.; Baccigalupi, C.; Ballardini, M.; Banday, A.J.; Barreiro, R.B.; Bartolo, N.; et al. Planck 2018 results. VI. Cosmological parameters. Astron. Astrophys. 2020, 641, A6.

13. Caldwell, R.R.; Dave, R.; Steinhardt, P.J. Cosmological imprint of an energy component with general equation of state. Phys. Rev. Lett. 1998, 80, 1582. [CrossRef]

14. Copeland, E.J.; Liddle, A.R.; Wands, D. Exponential potentials and cosmological scaling solutions. Phys. Rev. D 1998, 57, 4686. [CrossRef]

15. Tsujikawa, S. Quintessence: A Review. Class. Quant. Grav. 2013, 30, 214003. [CrossRef]

16. Armendariz-Picon, C.; Mukhanov, V.; Steinhardt, P.J. A Dynamical Solution to the Problem of a Small Cosmological Constant and Late-time Cosmic Acceleration. Phys. Rev. Lett. 2000, 85, 4438. [CrossRef] [PubMed]

17. Armendariz-Picon, C.; Mukhanov, V.; Steinhardt, P.J. Essentials of k-essence. Phys. Rev. D 2001, 63, 103510. [CrossRef]

18. Malquarti, M.; Copeland, E.J.; Liddle, A.R.; Trodden, M. A New view of k-essence. Phys. Rev. D 2003, 67, 123503. [CrossRef]

19. Scherrer, R.J. Purely kinetic k-essence as unified dark matter. Phys. Rev. Lett. 2004, 93, 011301. [CrossRef]

20. Sur, S.; Das, S. Multiple kinetic k-essence, phantom barrier crossing and stability. J. Cosmol. Astropart. Phys. 2009, 901, 007. [CrossRef]

21. Sur, S. Crossing the cosmological constant barrier with kinetically interacting double quintessence. arXiv 2009, arXiv:0902.1186.

22. Nojiri, S.; Odintsov, S.D. Introduction to modified gravity and gravitational alternative for dark energy. Int. J. Geom. Methods Mod. Phys. 2007, 4, 115-145. [CrossRef]

23. Tsujikawa, S. Modified gravity models of dark energy. Lect. Notes Phys. 2010, 800, 99-145.

24. Clifton, T.; Ferreira, P.G.; Padilla, A.; Skordis, C. Modified Gravity and Cosmology. Phys. Rep. 2012, 513, 1-189. [CrossRef] 
25. Papantonopoulos, E. Modifications of Einstein's Theory of Gravity at Large Distances; Lecture Notes in Physics; Springer: Cham, Switzerland, 2015.

26. Nojiri, S.; Odintsov, S.D.; Oikonomou, V.K. Modified Gravity Theories on a Nutshell: Inflation, Bounce and Late-time Evolution. Phys. Rep. 2017, 692, 1-104. [CrossRef]

27. Wand, L.; Steinhardt, P.J. Cluster abundance constraints for cosmological models with a time-varying, spatially inhomogeneous energy component with negative pressure. Astrophs. J. 1998, 508, 483.

28. Amendola, L. Coupled quintessence. Phys. Rev. D 2000, 62, 043511. [CrossRef]

29. Comelli, D.; Pietroni, M.; Riotto, A. Dark energy and dark matter. Phys. Lett. B 2003, 571, 115-120. [CrossRef]

30. Farrar, G.R.; Peebles, P.J.E. Interacting dark matter and dark energy. Astrophys. J. 2004, 604, 1. [CrossRef]

31. Cai, R.G.; Wang, A. Cosmology with interaction between phantom dark energy and dark matter and the coincidence problem. J. Cosmol. Astropart. Phys. 2005, 3, 002. [CrossRef]

32. Campo, S.; Herrera, R.; Olivares, G.; Pavon, D. Interacting models of soft coincidence. Phys. Rev. D 2006, 74, 023501. [CrossRef]

33. Amendola, L.; Appleby, S.; Avgoustidis, A.; Bacon, D.; Baker, T.; Baldi, M.; Bartolo, N.; Blanchard, A.; Bonvin, C.; Borgani, S.; et al. Cosmology and fundamental physics with the Euclid satellite. Living Rev. Relativ. 2018, 21, 2. [CrossRef] [PubMed]

34. Bertacca, D.; Bartolo, N.; Mataresse, S. Unified Dark Matter Scalar Field Models. Adv. Astron. 2010, 2010, 904379. [CrossRef]

35. Bertacca, D.; Bruni, M.; Piattella, O.F.; Pietrobon, D. Unified Dark Matter scalar field models with fast transition. J. Cosmol. Astropart. Phys. 2011, 1102, 018. [CrossRef]

36. Guendelman, E.; Nissimov, E.; Pacheva, S. Unified Dark Energy and Dust Dark Matter Dual to Quadratic Purely Kinetic K-Essence. Eur. Phys. J. C 2016, 76, 90. [CrossRef]

37. Ferreira, E.G.M.; Franzmann, G.; Khoury, J.; Brandenberger, R. Unified Superfluid Dark Sector. J. Cosmol. Astropart. Phys. 2019, 8, 027. [CrossRef]

38. Chamseddine, A.H.; Mukhanov, V.; Vikman, A. Cosmology with Mimetic Matter. J. Cosmol. Astropart. Phys. 2014, $1406,017$. [CrossRef]

39. Mirzagholi, L.; Vikman, A. Imperfect Dark Matter. J. Cosmol. Astropart. Phys. 2015, 1506, 028. [CrossRef]

40. Nojiri, S.; Odintsov, S.D.; Oikonomou, V.K. Viable Mimetic Completion of Unified Inflation-Dark Energy Evolution in Modified Gravity. Phys. Rev. D 2016, 94, 104050. [CrossRef]

41. Sebastiani, L.; Vagnozzi, S.; Myrzakulov, R. Mimetic gravity: A review of recent developments and applications to cosmology and astrophysics. Adv. High Energy Phys. 2017, 2017, 3156915. [CrossRef]

42. Langlois, D.; Mancarella, M.; Noui, K.; Vernizzi, F. Mimetic gravity as DHOST theories. J. Cosmol. Astropart. Phys. 2019 , 2, 036. [CrossRef]

43. Chemseddine, A.H.; Mukhanov, V. Ghost Free Mimetic Massive Gravity. J. High Energy Phys. 2018, 1806, 60. [CrossRef]

44. Chemseddine, A.H.; Mukhanov, V. Mimetic Massive Gravity: Beyond Linear Approximation. J. High Energy Phys. 2018, 1806, 62. [CrossRef]

45. Chamseddine, A.H.; Mukhanov, V.; Russ, T.B. Asymptotically Free Mimetic Gravity. Eur. Phys. J. C 2019, 79, 558. [CrossRef]

46. Chothe, H.R.; Dutta, A.; Sur, S. Cosmological Dark sector from a Mimetic-Metric-Torsion perspective. Int. J. Mod. Phys. D 2019, 28, 1950174. [CrossRef]

47. Sur, S.; Dutta, A.; Chothe, H.R. Mimetic-Metric-Torsion with induced Axial mode and Phantom barrier crossing. Eur. Phys. J. C 2021, 81, 306. [CrossRef]

48. Fujii, Y.; Maeda, K. The Scalar-Tensor Theory of Gravitation; Cambridge Monographs on Mathematical Physics, Cambridge University Press: Cambridge, UK, 2003.

49. Faraoni, V. Cosmology in Scalar-Tensor Gravity; Kluwer Academic Publishers: Dordrecht, The Netherlands, 2004.

50. Bartolo, N.; Pietroni, M. Scalar-tensor gravity and quintessence. Phys. Rev. D 2000, 61, 023518. [CrossRef]

51. Boisseau, B.; Esposito-Farese, G.; Polarski, D.; Starobinsky, A.A. Reconstruction of a scalar tensor theory of gravity in an accelerating universe. Phys. Rev. Lett. 2000, 85, 2236. [CrossRef] [PubMed]

52. Tsujikawa, S.; Uddin, K.; Mizuno, S.; Tavakol, R.; Yokoyama, J.I. Constraints on scalar-tensor models of dark energy from observational and local gravity tests. Phys. Rev. D 2008, 77, 103009. [CrossRef]

53. Elizalde, E.; Nojiri, S.; Odintsov, S.D. Late-time cosmology in (phantom) scalar-tensor theory: Dark energy and the cosmic speed-up. Phys. Rev. D 2004, 70, 043538. [CrossRef]

54. Campo, S.; Herrera, R.; Labrana, P. Emergent universe in a Jordan-Brans-Dicke theory. J. Cosmol. Astropart. Phys. 2007, $0711,030$. [CrossRef]

55. Boisseau, B.; Giacomini, H.; Polarski, D. Bouncing Universes in Scalar-Tensor Gravity Around Conformal Invariance. J. Cosmol. Astropart. Phys. 2016, 1605, 048. [CrossRef]

56. Saridakis, E.N.; Tsoukalas, M. Cosmology in new gravitational scalar-tensor theories. Phys. Rev. D 2016, 93, 124032. [CrossRef]

57. Kase, R.; Tsujikawa, S. Weak cosmic growth in coupled dark energy with a Lagrangian formulation. Phys. Lett. B 2020, 804, 135400. [CrossRef]

58. Koivisto, T. Growth of perturbations in dark matter coupled with quintessence. Phys. Rev. D 2005, 72, 043516. [CrossRef]

59. di Porto, C.; Amendola, L. Observational constraints on the linear fluctuation growth rate. Phys. Rev. D 2008, 77, 083508. [CrossRef]

60. Polarski, D.; Gannouji, R. On the growth of linear perturbations. Phys. Lett. B 2008, 660, 439. [CrossRef] 
61. Gannouji, R.; Polarski, D. The growth of matter perturbations in some scalar-tensor DE models. J. Cosmol. Astropart. Phys. 2008, 5, 018. [CrossRef]

62. $\mathrm{Wu}, \mathrm{P} . ; \mathrm{Yu}, \mathrm{H} . ; \mathrm{Fu}, \mathrm{X}$. A parametrization for the growth index of linear matter perturbations. J. Cosmol. Astropart. Phys. 2009, 6, 019. [CrossRef]

63. di Porto, C.; Amendola, L.; Branchini, E. Growth factor and galaxy bias from future redshift surveys: A study on parametrizations Mon. Not. Roy. Astron. Soc. 2011, 419, 985-997. [CrossRef]

64. Belloso, A.B.; Garcia-Bellido, J.; Sapone, D. A parametrization of the growth index of matter perturbations in various Dark Energy models and observational prospects using a Euclid-like survey. J. Cosmol. Astropart. Phys. 2011, 10, 010. [CrossRef]

65. Basilakos, S.; Pouri, A. The growth index of matter perturbations and modified gravity. Mon. Not. Roy. Astron. Soc. 2012, 423, 3761-3767. [CrossRef]

66. Steigerwald, H.; Bel, J.; Marinoni, C. Probing non-standard gravity with the growth index: A background independent analysis. J. Cosmol. Astropart. Phys. 2014, 5, 042. [CrossRef]

67. Batista, R.C. The impact of dark energy perturbations on the growth index. Phys. Rev. D 2014, 89, 123508. [CrossRef]

68. Malekjani, M.; Basilakos, S.; Mehrabi, A.; Davari, Z.; Rezaei, M. Agegraphic dark energy: Growth index and cosmological implications. Mon. Not. Roy. Astron. Soc. 2016, 464, 1192-1201. [CrossRef]

69. Polarski, D.; Starobinsky, A.A.; Giacomini, H. When is the growth index constant? J. Cosmol. Astropart. Phys. 2016, $12,037$. [CrossRef]

70. Basilakos, S.; Anagnostopoulos, F.K. Growth index of matter perturbations in the light of Dark Energy Survey. Eur. Phys. J. C 2020, 80, 212. [CrossRef]

71. Arjona, R.; García-Bellido, J.; Nesseris, S. Cosmological constraints on non-adiabatic dark energy perturbations. Phys. Rev. D 2020, 102, 103526 [CrossRef]

72. Basilakos, S.; Nesseris, S.; Perivolaropoulos, L. Observational constraints on viable $\mathrm{f}(\mathrm{R})$ parametrizations with geometrical and dynamical probes. Phys. Rev. D 2013, 87, 123529. [CrossRef]

73. Sur, S.; Bhatia, A.S. Weakly dynamic dark energy via metric-scalar couplings with torsion. J. Cosmol. Astropart. Phys. 2017, 1707, 039. [CrossRef]

74. Bhatia, A.S.; Sur, S. Dynamical system analysis of dark energy models in scalar coupled metric-torsion theories. Int. J. Mod. Phys. D 2017, 26, 1750149. [CrossRef]

75. Bhatia, A.S.; Sur, S. Phase Plane Analysis of Metric-Scalar Torsion Model for Interacting Dark Energy. arXiv 2016, arXiv:1611.06902.

76. Buchbinder, I.L.; Odintsov, S.D.; Shapiro, I.L. Nonsingular Cosmological Model with Torsion induced by Vacuum Quantum effects. Phys. Lett. B 1985, 162, 92-96. [CrossRef]

77. Buchbinder, I.L.; Odintsov, S.D.; Shapiro, I.L. Effective Action in Quantum Gravity; IOP: Bristol, UK, 1992.

78. Helayël-Neto, J.A.; Penna-Firme, A.; Shapiro, I.L. Conformal symmetry, anomaly and effective action for metric-scalar gravity with torsion. Phys. Lett. B 2000, 479, 411-420. [CrossRef]

79. Fonseca-Neto, J.B.; Romero, C.; Martinez, S.P.G. Scalar torsion and a new symmetry of general relativity. Gen. Relativ. Grav. 2013, 45, 1579-1601. [CrossRef]

80. Shapiro, I.L. Physical aspects of the space-time torsion. Phys. Rep. 2002, 357, 113-213. [CrossRef]

81. Einstein, A. The Meaning of Relativity: Including the Relativistic Theory of the Non-Symmetric Field, 5th ed.; Princeton University Press: Princeton, NJ, USA, 1970.

82. Trautman, A. Spin and torsion may avert gravitational singularities. Nature 1973, 242, 7-8. [CrossRef]

83. Hehl, F.W.; von der Heyde, P.; Kerlick, G.; Nester, J. General Relativity with Spin and Torsion: Foundations and Prospects. Rev. Mod. Phys. 1976, 48, 393. [CrossRef]

84. Raychaudhuri, A.K. Theoretical Cosmology; Clarendon Press: Oxford, UK, 1979.

85. de Sabbata, V.; Gasperini, M. Introduction to Gravitation; World Scientific: Singapore, 1985.

86. de Sabbata, V.; Sivaram, C. Spin Torsion and Gravitation; World Scientific: Singapore, 1994.

87. Hehl, F.W.; Obukhov, Y.N. How does the electromagnetic field couple to gravity, in particular to metric, nonmetricity, torsion and curvature? Lect. Notes Phys. 2001, 562, 479-504.

88. Blagojevic, M. Gravitation and Gauge Symmetries; IOP Publishing: London, UK, 2002.

89. Capozziello, S.; de Laurentis, M. Extended Theories of Gravity. Phys. Rep. 2011, 509, 167-321. [CrossRef]

90. Poplawski, N. Affine theory of gravitation. Gen. Relativ. Grav. 2014, 46, 1625. [CrossRef]

91. Westman, H.F.; Zlosnik, T.G. An introduction to the physics of Cartan gravity. Ann. Phys. 2015, 361, 330-376. [CrossRef]

92. Capozziello, S.; Lambiase, G.; Stornaiolo, C. Geometric classification of the torsion tensor of space-time. Ann. Phys. 2001, 10, 713-727. [CrossRef]

93. Bloomer, I. A maximally symmetric space with torsion. Gen. Relativ. Grav. 1978, 9, 763-771. [CrossRef]

94. Tsamparlis, M. Cosmological principle and torsion. Phys. Lett. A 1979, 75, 27. [CrossRef]

95. Gangopadhyay, D.; SenGupta, S. The meaning of Maximal Symmetry in presence of Torsion. arXiv 1997, arXiv:hep-th/9710139.

96. Gangopadhyay, D.; SenGupta, S. Duality invariance of cosmological solutions with torsion. Int. J. Mod. Phys. A 1999, 14, 4953-4966. [CrossRef]

97. Sur, S.; Bhatia, A.S. Constraining torsion in maximally symmetric (sub)spaces. Class. Quant. Grav. 2014, 31, 025020. [CrossRef] 
98. Majumdar, P.; SenGupta, S. Parity violating gravitational coupling of electromagnetic fields. Class. Quant. Grav. 1999, 16, L89. [CrossRef]

99. Hammond, R.T. Strings in gravity with torsion. Gen. Relativ. Grav. 2000, 32, 2007-2019. [CrossRef]

100. SenGupta, S.; Sur, S. Spherically symmetric solutions of gravitational field equations in Kalb-Ramond background. Phys. Lett. $B$ 2001, 521, 350-356. [CrossRef]

101. Kar, S.; Majumdar, P.; SenGupta, S.; Sinha, A. Does a Kalb-Ramond field make space-time optically active? Eur. Phys. J. C 2002, 23, 357-361. [CrossRef]

102. Kar, S.; Majumdar, P.; SenGupta, S.; Sur, S. Cosmic optical activity from an inhomogeneous Kalb-Ramond field. Class. Quant. Grav. 2002, 19, 677. [CrossRef]

103. Kar, S.; SenGupta, S.; Sur, S. Static spherisymmetric solutions, gravitational lensing and perihelion precession in Einstein-KalbRamond theory. Phys. Rev. D 2003, 67, 044005. [CrossRef]

104. SenGupta, S.; Sur, S. Does curvature dilaton coupling with a Kalb-Ramond field lead to an accelerating universe? J. Cosmol. Astropart. Phys. 2003, 312, 001. [CrossRef]

105. Maity, D.; SenGupta, S.; Sur, S. Spinning test particle in Kalb-Ramond background. Eur. Phys. J. C 2005, 42, 453. [CrossRef]

106. Sur, S.; Das, S.; SenGupta, S. Charged black holes in generalized dilaton-axion gravity. J. High Energy Phys. 2005, 510, 064. [CrossRef]

107. de Cesare, M.; Mavromatos, N.E.; Sarkar, S. On the possibility of tree-level leptogenesis from Kalb-Ramond torsion background. Eur. Phys. J. C 2015, 75, 514. [CrossRef] [PubMed]

108. Mukhopadhyaya, B.; Sen, S.; SenGupta, S. Does a Randall-Sundrum scenario create the illusion of a torsion free universe? Phys. Rev. Lett. 2002, 89, 121101; Erratum in 2002, 89, 259902. [CrossRef] [PubMed]

109. SenGupta, S.; Sur, S. Gravitational Redshift in Einstein-Kalb-Ramond space-time and Randall-Sundrum scenario. Europhys. Lett. 2004, 65, 601. [CrossRef]

110. Maity, D.; SenGupta, S.; Sur, S. Observable signals in a string inspired axion-dilaton background and Randall-Sundrum scenario. Phys. Rev. D 2005, 72, 066012. [CrossRef]

111. Mukhopadhyaya, B.; Sen, S.; SenGupta, S. A Randall-Sundrum scenario with bulk dilaton and torsion. Phys. Rev. D 2009, 79, 124029. [CrossRef]

112. Bhattacharjee, S.; Chatterjee, A. Gauge invariant coupling of fields to torsion: A string inspired model. Phys. Rev. D 2011, 8 , 106007. [CrossRef]

113. Das, A.; Mukhopadhyaya, B.; SenGupta, S. Why has spacetime torsion such negligible effect on the Universe? Phys. Rev. D 2014, 90, 107901. [CrossRef]

114. Hojman, S.; Rosenbaum, M.; Ryan, M.P. Propagating torsion and gravitation. Phys. Rev. D 1979, 19, 430. [CrossRef]

115. Carroll, S.M.; Field, G.B. Consequences of propagating torsion in connection dynamic theories of gravity. Phys. Rev. D 1994, 50, 3867. [CrossRef]

116. Saa, A. Propagating torsion from first principles. Gen. Relativ. Grav. 1997, 29, 205-220. [CrossRef]

117. Belyaev, A.S.; Shapiro, I.L. The action for the (propagating) torsion and the limits on the torsion parameters from present experimental data. Phys. Lett. B 1998, 425, 246-254. [CrossRef]

118. Hojman, R.; Mukku, C.; Sayed, W.A. Parity violation in metric torsion theories of gravitation. Phys. Rev. D 1980, $22,1915$. [CrossRef]

119. Mukhopadhyaya, B.; SenGupta, S.; Sur, S. Space-time torsion and parity violation: A gauge invariant formulation. Mod. Phys. Lett. A 2002, 17, 43-49. [CrossRef]

120. Mukhopadhyaya, B.; Sen, S.; SenGupta, S.; Sur, S. Parity violation and torsion: A study in four-dimensions and higher dimensions. Eur. Phys. J. C 2004, 35, 129. [CrossRef]

121. Maity, D.; Majumdar, P.; SenGupta, S. Parity violating Kalb-Ramond-Maxwell interactions and CMB anisotropy in a brane world. J. Cosmol. Astropart. Phys. 2004, 406, 005. [CrossRef]

122. Freidel, L.; Minic, D.; Takeuchi, T. Quantum gravity, torsion, parity violation and all that. Phys. Rev. D 2005, 72, 104002. [CrossRef]

123. Mercuri, S. From the Einstein-Cartan to the Ashtekar-Barbero canonical constraints, passing through the Nieh-Yan functional. Phys. Rev. D 2008, 77, 024036. [CrossRef]

124. Banerjee, K. Some Aspects of Holst and Nieh-Yan Terms in General Relativity with Torsion. Class. Quant. Grav. 2010, $27,135012$. [CrossRef]

125. Shapiro, I.L.; Teixeira, P.M. Quantum Einstein-Cartan theory with the Holst term. Class. Quant. Grav. 2014, 31, 185002. [CrossRef]

126. Hehl, F.W.; Obukhov, Y.N.; Rubilar, G.F.; Blagojevic, M. On the theory of the skewon field: From electrodynamics to gravity. Phys. Lett. A 2005, 347, 14. [CrossRef]

127. Rubilar, G.F.; Obukhov, Y.N.; Hehl, F.W. Torsion nonminimally coupled to the electromagnetic field and birefringence. Class. Quant. Grav. 2003, 20, L185. [CrossRef]

128. Ni, W.-T. Skewon field and cosmic wave propagation. Phys. Lett. A 2014, 378, 1217-1223. [CrossRef]

129. Yo, H.-J.; Nester, J.M. Dynamic Scalar Torsion and an Oscillating Universe. Mod. Phys. Lett. A 2007, 22, 2057-2069. [CrossRef]

130. Shie, K.-F.; Nester, J.M.; Yo, H.-J. Torsion Cosmology and the Accelerating Universe. Phys. Rev. D 2008, 78, 023522. [CrossRef]

131. Baekler, P.; Hehl, F.W.; Nester, J.M. Poincaré gauge theory of gravity: Friedmann cosmology with even and odd parity modes. Analytic part. Phys. Rev. D 2011, 83, 024001. [CrossRef] 
132. Blagojevic, M.; Hehl, F.W. Gauge Theories of Gravitation: A Reader with Commentaries; World Scientific: Singapore, 2013.

133. Lu, J.; Chee, G. Cosmology in Poincaré gauge gravity with a pseudoscalar torsion. J. High Energy Phys. 2016, 1605, 024. [CrossRef]

134. Nikiforova, V.; Randjbar-Daemi, S.; Rubakov, V. Self-accelerating Universe in modified gravity with dynamical torsion. Phys. Rev. D 2017, 95, 024013. [CrossRef]

135. Bengochea, G.R.; Ferraro, R. Dark torsion as the cosmic speed-up. Phys. Rev. D 2009, 79, 124019. [CrossRef]

136. Li, B.; Sotiriou, T.P.; Barrow, J.D. Large-scale Structure in $f(T)$ Gravity. Phys. Rev. D 2011, 83, 104017. [CrossRef]

137. Cai, Y.-F.; Chen, S.-H.; Dent, J.B.; Dutta, S.; Saridakis, E.N. Matter Bounce Cosmology with the $f(T)$ Gravity. Class. Quant. Grav. 2011, 28, 215011. [CrossRef]

138. Geng, C.-Q.; Lee, C.-C.; Saridakis, E.N.; Wu, Y.-P. “Teleparallel” dark energy. Phys. Lett. B 2011, 704, 384. [CrossRef]

139. Cai, Y.-F.; Capozziello, S.; de Laurentis, M.; Saridakis, E.N. $f(T)$ teleparallel gravity and cosmology. Rep. Prog. Phys. 2016, 79, 106901. [CrossRef] [PubMed]

140. Bahamonde, S.; Capozziello, S.; Faizal, M.; Nunes, R.C. Nonlocal Teleparallel Cosmology. Eur. Phys. J. C 2017, 77, 628. [CrossRef] [PubMed]

141. Vignolo, S.; Fabbri, L.; Stornaiolo, C. A square-torsion modification of Einstein-Cartan theory. Ann. Phys. 2012, 524, 826. [CrossRef]

142. Vasilev, T.B.; Cembranos, J.A.R.; Valcarcel, J.G.; Martín-Moruno, P. Stability in quadratic torsion theories. Eur. Phys. J. C 2017, 77, 755. [CrossRef]

143. Kaul, R.K.; Sengupta, S. Degenerate spacetimes in first order gravity. Phys. Rev. D 2016, 93, 084026. [CrossRef]

144. Flanagan, E.E.; Rosenthal, E. Can gravity probe B usefully constrain torsion gravity theories? Phys. Rev. D 2007, 75, 124016. [CrossRef]

145. Kostelecky, V.A.; Russell, N.; Tasson, J. New Constraints on Torsion from Lorentz Violation. Phys. Rev. Lett. 2008, 100, 111102. [CrossRef] [PubMed]

146. Babourova, O.V.; Frolov, B.N. Interaction of the 4-rotational gauge field with orbital momentum, gravidiamagnetic effect and orbit experiment 'Gravity Probe B'. Phys. Rev. D 2010, 82, 027503. [CrossRef]

147. Hehl, F.W.; Obukhov, Y.N.; Puetzfeld, D. On Poincaré gauge theory of gravity, its equations of motion and Gravity Probe B. Phys. Lett. A 2013, 377, 1775. [CrossRef]

148. Camera, S.; Cardone, V.F.; Radicella, N. Detectability of Torsion Gravity via Galaxy Clustering and Cosmic Shear Measurements. Phys. Rev. D 2014, 89, 083520. [CrossRef]

149. Castillo-Felisola, O.; Corral, C.; Schmidt, I.; Zerwekh, A.R. Updated limits on extra dimensions through torsion and LHC data. Mod. Phys. Lett. A 2014, 29, 1450081. [CrossRef]

150. Lucat, S.; Prokopec, T. Observing Geometrical Torsion. arXiv 2017, arXiv:1705.00889.

151. Jacobson, T.; Mattingly, D. Gravity with a dynamical preferred frame. Phys. Rev. D 2001, 64, 024028. [CrossRef]

152. Jacobson, T.; Mattingly, D. Einstein-Aether waves. Phys. Rev. D 2004, 70, 024003. [CrossRef]

153. Jacobson, T.; Liberati, S.; Mattingly, D. Lorentz violation at high energy: Concepts, phenomena and astrophysical constraints. Ann. Phys. 2006, 321, 150-196. [CrossRef]

154. Ryan, J.; Doshi, S.; Ratra, B. Constraints on dark energy dynamics and spatial curvature from Hubble parameter and baryon acoustic oscillation data. Mon. Not. R. Astron. Soc. 2018, 480, 759-767. [CrossRef]

155. Gonzalez, J.E.; Alcaniz, J.S.; Carvalho, J.C. Non-parametric reconstruction of cosmological matter perturbations. J. Cosmol. Astropart. Phys. 2016, 2016, 016. [CrossRef]

156. Benisty, D.; Guendelman, E.I.; Nissimov, E.; Pacheva, S. $\Lambda$ CDM as a Noether Symmetry in Cosmology. arXiv 2020, arXiv:2003.13146.

157. Calderon, R.; Felbacq, D.; Gannouji, R.; Polarski, D.; Starobinsky, A.A. Global properties of the growth index of matter inhomogeneities in the Universe. Phys. Rev. D 2019, 100, 083503. [CrossRef]

158. Khyllep, W.; Dutta, J. Linear growth index of matter perturbations in Rastall gravity. Phys. Lett. B 2019, 797, 134796. [CrossRef]

159. Tsujikawa, S.; Gannouji, R.; Moraes, B.; Polarski, D. Dispersion of growth of matter perturbations in $\mathrm{f}(\mathrm{R})$ gravity. Phys. Rev. D 2009, 80, 084044. [CrossRef]

160. Beutler, F.; Blake, C.; Colless, M.; Jones, D.H.; Staveley-Smith, L.; Poole, G.B.; Campbell, L.; Parker, Q.; Saunders, W.; et al. The $6 \mathrm{dF}$ Galaxy Survey: $z \approx 0$ measurements of the growth rate and $\sigma_{8}$. Mon. Not. R. Astron. Soc. 2012, 423, 3430-3444. [CrossRef]

161. Ata, M.; Baumgarten, F.; Bautista, J.; Beutler, F.; Bizyaev, D.; Blanton, M.R.; Blazek, J.A.; Bolton, A.S.; Brinkmann, J.; Brownstein, J.R.; et al. The clustering of the SDSS-IV extended Baryon Oscillation Spectroscopic Survey DR14 quasar sample: first measurement of baryon acoustic oscillations between redshift 0.8 and 2.2. Mon. Not. R. Astron. Soc. 2017, 473, 4773-4794. [CrossRef]

162. Beutler, F.; Seo, H.J.; Saito, S.; Chuang, C.H.; Cuesta, A.J.; Eisenstein, D.J.; Gil-Marín, H.; Grieb, J.N.; Hand, N.; Kitaura, F.S.; et al. The clustering of galaxies in the completed SDSS-III Baryon Oscillation Spectroscopic Survey: anisotropic galaxy clustering in Fourier space. Mon. Not. R. Astron. Soc. 2016, 466, 2242-2260. [CrossRef]

163. Sánchez, A.G.; Montesano, F.; Kazin, E.A.; Aubourg, E.; Beutler, F.; Brinkmann, J.; Brownstein, J.R.; Cuesta, A.J.; Dawson, K.S.; Eisenstein, D.J.; et al. The clustering of galaxies in the SDSS-III Baryon Oscillation Spectroscopic Survey: Cosmological implications of the full shape of the clustering wedges in the data release 10 and 11 galaxy samples. Mon. Not. R. Astron. Soc. 2014, 440, 2692-2713. [CrossRef] 
164. Feix, M.; Nusser, A.; Branchini, E. Growth rate of cosmological perturbations at $z \sim 0.1$ from a new observational test. Phys. Rev. Lett. 2015, 115, 011301. [CrossRef] [PubMed]

165. Blake, C.; Baldry, I.K.; Bland-Hawthorn, J.; Christodoulou, L.; Colless, M.; Conselice, C.; Driver, S.P.; Hopkins, A.M.; Liske, J.; Loveday, J.; et al. Galaxy In addition, Mass Assembly (GAMA): improved cosmic growth measurements using multiple tracers of large-scale structure. Mon. Not. Roy. Astron. Soc. 2013, 436, 3089-3105. [CrossRef]

166. Song, Y.S.; Percival, W.J. Reconstructing the history of structure formation using redshift distortions. J. Cosmol. Astropart. Phys. 2009, 10, 004. [CrossRef]

167. Shi, F.; Yang, X.; Wang, H.; Zhang, Y.; Mo, H.J.; van den Bosch, F.C.; Luo, W.; Tweed, D.; Li, S.; Liu, C.; Lu, Y. Mapping the Real Space Distributions of Galaxies in SDSS DR7: II. Measuring the growth rate, linear mass variance and biases of galaxies at redshift 0.1. arXiv 2017, arXiv:1712.04163.

168. Howlett, C.; Staveley-Smith, L.; Elahi, P.J.; Hong, T.; Jarrett, T.H.; Jones, D.H.; Koribalski, B.S.; Macri, L.M.; Masters, K.L.; Springob, C.M. 2MTF-VI. Measuring the velocity power spectrum. Mon. Not. R. Astron. Soc. 2017, 471, 3135-3151. [CrossRef]

169. De La Torre, S.; Jullo, E.; Giocoli, C.; Pezzotta, A.; Bel, J.; Granett, B.R.; Guzzo, L.; Garilli, B.I.; Scodeggio, M.; Bolzonella, M.; et al. The VIMOS Public Extragalactic Redshift Survey (VIPERS)-Gravity test from the combination of redshift-space distortions and galaxy-galaxy lensing at $0.5<z<1.2$. Astron. Astrophys. 2017, 608, A44.

170. Gil-Marín, H.; Percival, W.J.; Verde, L.; Brownstein, J.R.; Chuang, C.H.; Kitaura, F.S.; Rodríguez-Torres, S.A.; Olmstead, M.D. The clustering of galaxies in the SDSS-III Baryon Oscillation Spectroscopic Survey: RSD measurement from the power spectrum and bispectrum of the DR12 BOSS galaxies. Mon. Not. R. Astron. Soc. 2016, 465, 1757-1788. [CrossRef]

171. Samushia, L.; Percival, W.J.; Raccanelli, A. Interpreting large-scale redshift-space distortion measurements. Mon. Not. R. Astron. Soc. 2012, 420, 2102-2119. [CrossRef]

172. Kazantzidis, L.; Perivolaropoulos, L.; Skara, F. Constraining power of cosmological observables: Blind redshift spots and optimal ranges. Phys. Rev. D 2019, 99, 063537. [CrossRef]

173. Nesseris, S.; Pantazis, G.; Perivolaropoulos, L. Tension and constraints on modified gravity parametrizations of $G_{e f f}(z)$ from growth rate and Planck data. Phys. Rev. D 2017, 96, 023542. [CrossRef]

174. D'Agostino, R.; Luongo, O. Growth of matter perturbations in nonminimal teleparallel dark energy. Phys. Rev. D 2018, 98, 124013. [CrossRef]

175. L'Huillier, B.; Shafieloo, A.; Kim, H. Model-independent cosmological constraints from growth and expansion. Mon. Not. R. Astron. Soc. 2018, 476, 3263-3268. [CrossRef]

176. Kazantzidis, L.; Perivolaropoulos, L. Evolution of the $\sigma_{8}$ tension with the Planck $15 / \Lambda C D M$ determination and implications for modified gravity theories. Phys. Rev. D 2018, 97, 103503. [CrossRef] 